\title{
Percepciones del docente universitario sobre la comunicación científica de libre acceso
}

\author{
Alba Ruth Pinto-Santos*, Carlos Villanueva-Valadez **, Omar Cortés-Peña*** \\ * Facultad Ciencias de la Educación. Universidad de la Guajira. Colombia. \\ Correo-e: arpinto@uniguajira.edu.co | ORCID iD: https://orcid.org/0000-0001-8414-544X \\ ** Facultad Ciencias de la Comunicación. Universidad Autónoma de Nuevo León. México. \\ Correo-e: carlos.villanuevavl@uanl.edu.mx| ORCID iD: https://orcid.org/0000-0001-6914-4780 \\ *** Facultad de Psicología. Universidad Sergio Arboleda. Colombia. \\ Correo-e: omar.cortes@correo.usa.edu.co | ORCID iD: https://orcid.org/0000-0002-9603-885X
}

Recibido: 18-05-2018; 2a versión: 05-09-2018; Aceptado: 13-09-2018.

Cómo citar este artículo/Citation: Pinto-Santos, A. R.; Villanueva-Valadez, C.; Cortés-Peña, O. (2019). Percepciones del docente universitario sobre la comunicación científica de libre acceso. Revista Española de Documentación Científica, 42 (2): e233. https://doi.org/10.3989/redc.2019.2.1588

\begin{abstract}
Resumen: Internet ha generado cambios en la comunicación científica creando un nuevo modelo de acceso libre y rápido a los resultados de investigación al que poco a poco se adaptan las instituciones educativas. Este artículo analiza la percepción sobre políticas y prácticas de acceso abierto por docentes de La Universidad de la Guajira - Colombia (Uniguajira) y la Universidad Autónoma de Nuevo León- México (UANL). Metodológicamente se asumió una investigación no experimental de corte comparativo. Se utilizó un diseño muestral aleatorio con (n: 245) participantes. Los resultados evidencian una actitud favorable de los docentes frente a la comunicación científica en abierto, con valoraciones superiores en la escala media general de 3.3/4 para UANL frente a 2.97/4 en Uniguajira. Se concluye que existe disposición de los docentes para compartir contenidos científicos de libre acceso, y que a nivel institucional se debe fortalecer políticas y prácticas que favorezcan el acceso abierto.
\end{abstract}

Palabras clave: acceso abierto; repositorios institucionales; revistas científicas; comunicación científica; políticas de acceso abierto, universidades.

\section{Perceptions of the university professor on the open access scientific communication}

Abstract: The Internet have generated changes in scientific communication, creating a new model of free and quick access to research results to which educational institutions are gradually adapting in different contexts. This article analyzes the perception of open access policies and practices by teachers of the Uniguajira - Colombia (Uniguajira) and the Universidad Autónoma de Nuevo León - Mexico (UANL). Methodologically, a comparative, non-experimental investigation was assumed. A random sample design with ( $\mathrm{n}: 245)$ participants was used. The results show a favorable attitude of teachers towards open scientific communication, with higher ratings in the general average scale of 3.3 / 4 for UANL compared to $2.97 / 4$ in Uniguajira. It is concluded that there is a willingness on part of teachers to share scientific content in free access; nonetheless, at the institutional level, open access policies and practices need to be strengthened.

Keywords: open access; institutional repositories; scientific magazines; scientific communication; open access policies, universities.

Copyright: (c) 2019 CSIC. Este es un artículo de acceso abierto distribuido bajo los términos de la licencia de uso y distribución Creative Commons Reconocimiento 4.0 Internacional (CC BY 4.0). 


\section{INTRODUCCIÓN}

Open Access o Acceso Abierto es definido como la disponibilidad libre y gratuita de contenidos científicos y académicos en internet (Abadal, 2013; Serna-Saucedo y Villanueva-Valadez, 2014). Implica que el autor autorice a usar, descargar, compartir y difundir información científica en cualquier formato (Swan, 2013). Asimismo, el acceso abierto es un cambio en la forma de comunicación científica que promueve la difusión y reutilización del conocimiento (Arévalo y otros, 2008; Abadal, 2013), desde los principios de libertad, flexibilidad e imparcialidad (Swan, 2013).

Por consiguiente, para que los contenidos científicos sean de acceso abierto deben ser gratuitos y de acceso libre (Abadal, 2013). Es decir, que se puede llegar a ellos sin ningún costo y se tiene la libertad para descargarlos y distribuirlos, reconociendo los derechos de autor. El objetivo del acceso abierto es mejorar la comunicación científica y eliminar las barreras en el acceso a la información (Arévalo y otros, 2008). Asimismo, a partir de un acelerado proceso de investigaciones donde se conocen de manera rápida los avances que se están presentando, la producción científica incrementa la eficiencia a través del acceso abierto a las ideas y a mayor audiencia (Canessa y Zennaro, 2008).

De igual manera, el acceso abierto viabiliza la superación de las llamadas brechas de la información frente a la producción científica entre países ricos (centro) y pobres (periferia), aumenta la capacidad de trabajo de los científicos de diferentes países, ayuda a dar mayor visibilidad de la producción científica de la periferia, favorece la publicación en revistas y repositorios, genera acceso libre a literatura científica y acceso a información de alto valor académico, y permite continuar avanzando en los proyectos de investigación que han realizado otros investigadores (Guédon, 2011). Es una forma de democratizar el conocimiento, donde la circulación de la comunicación científica no depende del idioma o de lo que se considere importante en algunos países (Vargas, 2014; AguadoLópez y Becerril-García, 2016).

\section{ANTECEDENTES}

La concepción de investigador que trabaja en solitario y que finalmente presenta a la comunidad científica sus descubrimientos está alejada de la dinámica actual, donde la colaboración científica es fundamental en la construcción de conocimiento (González-Alcaide y Gómez-Ferri, 2014; Cortés y otros, 2015; Aguado-López y Becerril-García, 2016). Es por ello que el movimiento acceso abierto, gestado por académicos y científicos que han creído en las ventajas que ofrece internet para la difusión de la ciencia abierta y generar colaboración en la comunidad académica, ha tenido aceptación y sigue creciendo. El acceso abierto tiene los principales antecedentes en la firma de la Declaración de Budapest (2002) que establece las vías de publicación, la Declaración de Bethesda (2003) que debate en torno a los derechos de autor y las condiciones de publicación, y la Declaración de Berlín (2003) que propone estímulos a los investigadores para publicar en esta modalidad.

En este sentido, es la misma comunidad académica y científica la que ha ido fortaleciendo el movimiento acceso abierto (Pinto y otros, 2017). Las ventajas del Open Access van más allá de la comunicación de la ciencia, dado que implica mayor uso e impacto de las publicaciones, incremento de la investigación desde la reutilización de datos y avances de otros científicos, disminución de costos en acceso a la literatura científica, acceso libre y transferencia del conocimiento a los individuos, reducción de brechas entre países al tener acceso a los mismos contenidos, y visibilización de la inversión pública en investigación (Abadal, 2013).

Existen dos vías para la publicación en acceso abierto: La vía dorada relacionada con la publicación en revistas científicas sin costo para los lectores, las cuales pueden ser especializadas o multidisciplinarias, con procesos de revisión externo (peer-review) o de divulgación. También existe la vía verde que está asociada al uso de los Repositorios Institucionales para administrar, preservar, visibilizar, y difundir la producción científica de las instituciones por medio de publicación en bases de datos de acceso libre (Abadal, 2013; Valverde, 2013; Serna-Saucedo y Villanueva-Valadez, 2014).

\section{Acceso Abierto en la educación}

En los últimos años se ha generado un aumento sostenible del movimiento de acceso abierto en universidades y gobiernos. Proyectos sobre la apertura de repositorios abanderados por la vía verde y el apoyo de la comunidad científica sugieren la necesidad de continuar impulsando este tipo de iniciativas a favor de la comunicación abierta y libre de la ciencia. Instituciones como Scholarly Publishing and Academic Resources Coalition (SPARC), Open Access Scholarly Publishers Association (OASPA) y Public Library of Science (PLOS) han contribuido para la prontitud de enriquecer la educación y la innovación mediante el acotamiento de las barreras al acceso del conocimiento (Del Rio Romero, 2014).

El interés por el acceso abierto ha provocado que académicos desarrollen diversos estudios sobre la implementación de esta filosofía, así como 
el desglose minucioso sobre los componentes que cada institución debe trabajar para su correcta implementación. Cabrera (2014) realiza una descripción de los modelos de acceso abierto en seis países pioneros del movimiento (Argentina, Australia, Brasil, Estados Unidos, Inglaterra y España) desde la perspectiva de la Unión Europea. Dicho análisis tiene su génesis desde el marco legal del acceso abierto, hasta la interrelación que poseen los modelos que fueron implementados en los países estudiados.

Si bien el acceso abierto es un fenómeno de mediación social debido a que actúa como un canal para la divulgación de la ciencia y el conocimiento, tiene que poseer un sustento legal como todo medio formal de transmisión de mensajes (libros, artículos, tesis, etc.). El mismo Cabrera (2014) contextualiza la importancia del marco legal del acceso abierto, fundamentando los cambios en las tecnologías de la información y comunicación (TIC). De igual manera, Arencibia (2006) afirma que las variaciones tecnológicas han extendido nuevas formas de compartir información, concibiendo brillantes proyectos que ambicionan el desarrollo de la cultura, la educación y la ciencia como aspectos imprescindibles para el crecimiento económico y social.

Peter Suber (2015) cita el caso de la Red de Revistas Científicas de América Latina y el Caribe, España y Portugal (Redalyc), agregando que este proyecto contribuye a la diseminación y visibilidad de la producción académica y científica proveniente de los países de habla hispana, además de constituirse como una de las principales fuentes de información cien por ciento confiable. Por esta razón, Redalyc es un modelo que guía la publicación científica para fortalecer los espacios incluyentes y colaborativos, de los cuales podemos matizar la creación de repositorios temáticos, institucionales y nacionales que garantizan la divulgación de conocimiento y que esté al alcance de todos.

A partir de lo anteriormente expuesto, y teniendo presente que la Uniguajira y la UANL han implementado políticas institucionales de acceso abierto al conocimiento, se hace necesario conocer la apreciación de los docentes sobre este tipo de modalidad de publicación y la forma como la universidad lo está promoviendo. Para ello este estudio plantea tres objetivos específicos de investigación, a saber: definir las percepciones de los docentes sobre la forma como su universidad administra la producción científica, reconocer la disposición del docente universitario por compartir información de manera libre y a través de internet, e identificar los conocimientos que tienen los profesores sobre los derechos de autor en modalidad acceso abierto.

\section{MÉTODO}

El presente estudio asumió las posturas del paradigma positivista desde los postulados de Valenzuela y Flores (2012), según el cual busca entender la realidad de manera objetiva. Desde esta perspectiva y en concordancia con León y Montero (2003) se abordó una investigación no experimental de corte comparativo. En tal sentido corresponde a un diseño no experimental dado que no se genera una manipulación de variables independientes, y es comparativo para efecto de analizar las similitudes y diferencias existentes entre los participantes de la Uniguajira (Colombia) y de UANL (México) frente a las percepciones sobre visibilización de la producción científica.

\subsection{Población y Muestra}

Para la selección de los participantes del estudio se establecieron los siguientes criterios de inclusión/exclusión:

- Ser docente tiempo completo de las universidades seleccionadas para el estudio: Que el docente estuviera vinculado a UANL (México) o Uniguajira (Colombia).

- Ser docente investigador: Que los docentes de las dos universidades estuvieran registrados en los respectivos centros de investigación y/o vinculados a proyectos de investigación.

- Que existiera participación de diferentes facultades y sedes de la Universidad: Tener información representativa de los diferentes programas y sedes de las respectivas universidades.

- Disponibilidad de participación: Que el docente aceptara participar de la investigación respondiendo al instrumento presentado a través de correo electrónico.

La selección de las dos universidades participantes se realizó con la intención de analizar las diferencias de las percepciones sobre políticas y prácticas de acceso abierto en la visibilización académica y científica que tienen los docentes de cada universidad, asumiendo que la UANL lleva más de 8 años impulsando políticas de acceso abierto y administrando los resultados de investigación a través de un repositorio institucional, y Uniguajira es nueva en ese proceso. Además, las dos Universidades participantes son financiadas por fondos públicos de los respectivos países, cuentan con diferentes sedes, y tienen una amplia oferta de programas académicos. Para el caso de la UANL es la tercera universidad más grande de México, la de mayor presencia en el noreste del país, con un alto reconocimiento de programas de pregrado y posgrado. Por su parte Uni- 
guajira es una institución relativamente joven, es la Institución de Educación Superior (IES) de mayor presencia en el departamento, y en los últimos años ha mostrado avances significativos en los procesos de categorización de los grupos de investigación adscritos al Departamento Administrativo de Ciencia, Tecnología e Innovación (Colciencias).

En este sentido, se tomó una muestra no probabilística de ( $n: 249)$ participantes, que está conformada por dos submuestras, Uniguajira $\left(n_{1}: 130\right)$ y UANL $\left(n_{2}: 119\right)$. Es decir, el $52 \%$ de la muestra lo aporta la primera universidad, mientras que el $47.8 \%$ la U de León, por lo tanto las muestras son muy cercanas teniendo presente el número de docentes registrados en la dirección de investigación de cada Universidad. En relación con la composición por género, en el caso de Uniguajira se distribuyeron de forma similar, es decir $50 \%$ hombres y $50 \%$ mujeres, y en UANL el $46,15 \%$ son hombres y $53,85 \%$ son mujeres, guardando balance en la participación. En relación con la edad de los participantes, el rango de menor participación son los profesores menores de 30 años con 6,1\% en Uniguajira y $6,5 \%$ en UANL, entre 31 y 40 años $32,3 \%$ y $39,5 \%$ respectivamente, de 41 a 50 años son también cercanas con $32,3 \%$ y $41,1 \%$ para cada universidad. Mientras que de 51 o más, hay $29,2 \%$ en Uniguajira y $14,2 \%$ en UANL. Esto significa que la muestra de Uniguajira tiene participantes de mayor edad.

De acuerdo con la participación por áreas de conocimiento, en el caso de Uniguajira la mayor representación la mostró la facultad de Ingeniería con $32,3 \%$, seguida de Ciencias de la Educación con $26,9 \%$, Ciencias Sociales y Humanas con 15,4\%, Ciencias Económicas 23,1\%, y Ciencias Básicas con 2,3\%. La UANL presentó participación de 16,0\% en Ingeniería, 6,7\% Ciencias de la Educación, 24,4\% Ciencias Sociales y Humanas, 5,9\% Ciencias Económicas, 15,1\% Ciencias Básicas, $26,1 \%$ Ciencias de la Salud y $5,9 \%$ Bellas Artes. Se buscó en todo caso tener participación de las diferentes facultades y programas, a través de la invitación y socialización del proyecto de investigación en las respectivas facultades de cada universidad.

\subsection{Técnicas e Instrumentos}

En el desarrollo del estudio, partiendo de los referentes teóricos citados en la introducción, los autores diseñaron el instrumento denominado Escala Actitudinal de Situaciones Asociadas a la Visibilización de la Producción Científica. La versión inicial del instrumento estaba conformada por 30 ítems, posteriormente fue sometido a validación por parte de (3) expertos con los criterios de relevancia y pertinencia frente a cada categoría de análisis. Los ítems que presentaron una valoración negativa por dos o más expertos fueron sustituidos o eliminados, por lo tanto, la versión final de la Escala Actitudinal de Situaciones Asociadas a la Visibilización de la Producción Científica quedó conformada por 22 ítems (cada uno con una afirmación y opciones de respuesta de 1 a 4 , siendo 4 una actitud más favorable). Las tres categorías de análisis del instrumento son las siguientes:

- Visibilización de la producción científica y académica de la universidad: Hace referencia al conocimiento que tienen los docentes sobre la producción académica y científica de la universidad, la formas como se puede acceder a dichos productos y la manera como administra los resultados de investigación.

- Importancia del repositorio institucional: Se relaciona con los conocimientos que tienen los docentes del repositorio institucional de la universidad y su disposición para compartir información a través de esta vía de la publicación en abierto.

- Derechos de autor y acceso abierto: Se relaciona con las falsas creencias que tienen los docentes sobre el acceso abierto, la protección de derechos autor, y las formas de comunicar la ciencia en la era digital.

La escala actitudinal de situaciones asociadas a la visibilización de la producción científica (Pinto y Villanueva, 2016), en términos de confiabilidad, después de recodificadas las preguntas negativas, es decir, que iban en un sentido diferente a la escala, presentó un nivel de confiabilidad Alfa de Cronbach de $(\alpha: 783)$. De igual manera, se pudo identificar que los rangos de intercorrelaciones entre la escala y las preguntas oscilan entre ( $r: .151)$ y ( $r: .449)$, por lo tanto, se evidencia que todos los ítems generaron correlación interna y positiva con la escala.

\subsection{Procedimiento}

En relación con el procedimiento de la investigación, el estudio se realizó en cuatro etapas. Primero se diseñó y validó el instrumento de recolección de datos a partir de la revisión de la literatura. Seguidamente, se aplicó la escala actitudinal de situaciones asociadas a la visibilización de la producción científica, la cual fue elaborada a través de la herramienta Formulario facilitada por el paquete Google del correo institucional. Éste instrumento fue enviado desde la dirección de investigación de cada universidad a través de correos electrónicos a la muestra seleccionada. Posteriormente se realizó el análisis de la información obtenida y se tabuló y organizó la información obtenida de las dos Instituciones Educativas participantes a través de soft- 
ware SPSS. Finalmente, se contrastó la información de cada universidad, estableciendo diferencias y puntos de encuentro. Esta etapa fue fundamental para comprender los resultados, las implicaciones de apropiar políticas y prácticas de acceso abierto, y generar conclusiones.

\section{RESULTADOS}

Al comparar el número de publicaciones científicas que registran los docentes ante la dirección de investigación desde el año 2014 al 2016, se encontró que en términos generales La UANL tiene mayor producción académica y científica (Véase figura 1). Se puede apreciar en la gráfica que con ninguna publicación aparece el 34,8\% para Uniguajira y solamente $5,8 \%$ para UANL. Con una, dos o tres publicaciones Uniguajira tiene la mayor cantidad de participación con el 17,0\%, 16,2\% y 14,7\%. Mientras que, con cuatro, cinco y seis o más publicaciones la UANL tiene los mejores márgenes de participación con el $26,0 \%, 21,8 \%$ y $26,0 \%$ respectivamente. Lo anterior evidencia un perfil en el que la UANL tiene mayor cantidad de producción académicas en comparación con Uniguajira.

A partir del análisis de los factores que atribuyen los docentes al interés de publicación, se encuentra para el caso de Uniguajira que la mayor motivación está relacionada con Visibilizar sus investigaciones con 44,6\%, guardando relación similar a la Universidad de Nuevo León con 47,1\% (Tabla I). No obstante, se encuentra que el ítem Exigencia laboral tiene para Uniguajira menor relevancia con $3,8 \%$, contrastando con la Universidad de Nuevo León que representa el 28,6\%.

Continuando con el análisis de la escala actitudinal de situaciones asociadas a la visibilización de la producción científica, es de aclarar que las puntuaciones promedio se calcularon para cada una de las (3) subescalas, a saber: Visibilidad de la producción científica (compuesta por los ítems 1, 2, 3, 4, 5, 6, 7,11 y 12), Importancia del repositorio institucional (conformada por los ítems 8, 9, 16, 17 y 22), y Derechos de autor (compuesta por los ítems 10, 13, $14,15,18,19,20$ y 21 ). Estos puntajes promedio de cada una de las ( 3 ) subescalas, al igual que para la Escala General fueron calculados a partir de las sumatorias de las puntuaciones de los ítems, divida entre el número de ítems de cada escala; por lo tanto los puntajes de las (3) subescalas y la escala general también quedaron comprendidos con un rango de (1 a 4). Cuanto más alto es el puntaje, se presenta un nivel de actitud más favorable en cada una de las categorías de análisis.

Figura 1. Comparación publicaciones científicas por universidad

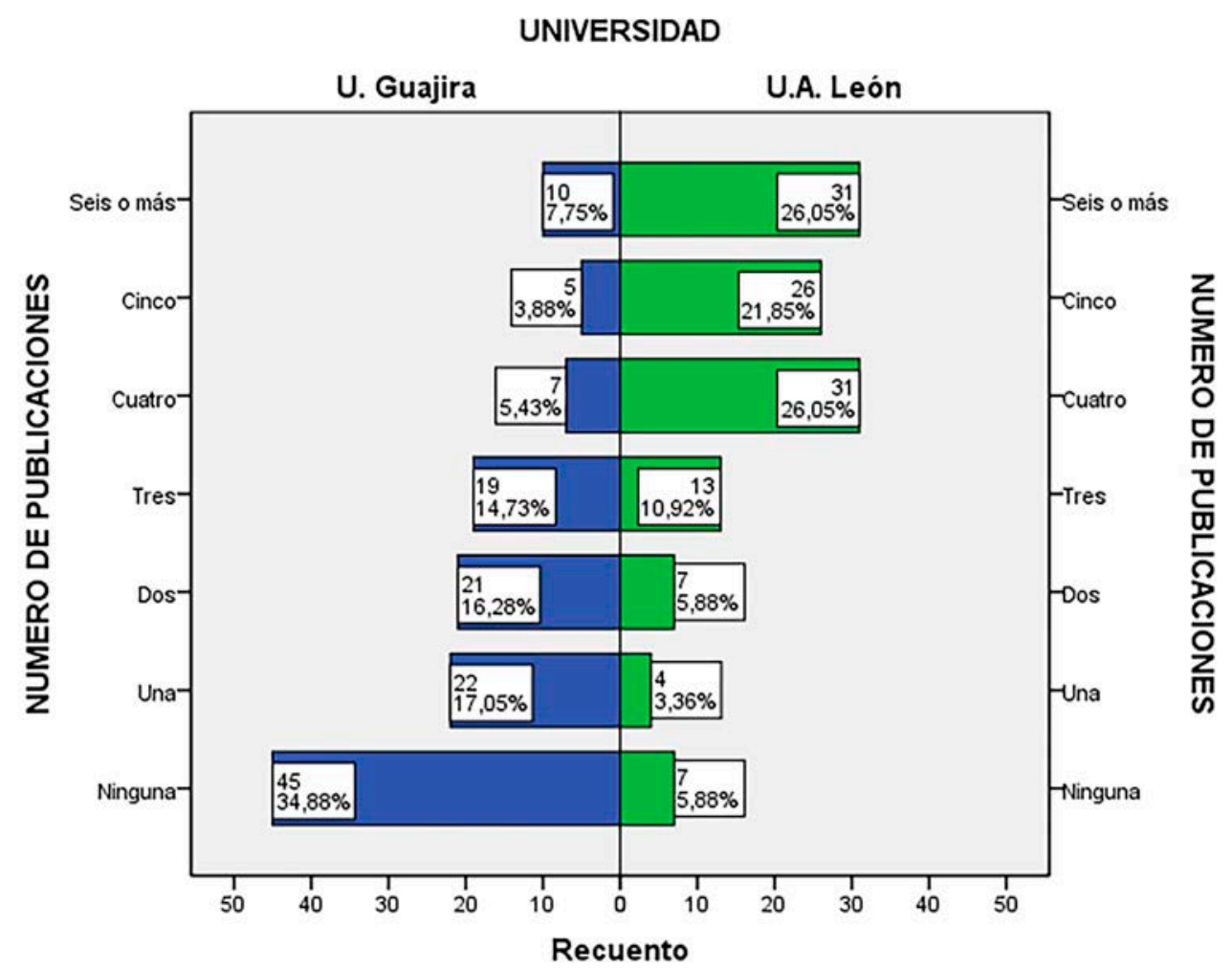


En términos de la producción científica el promedio general en una escala de (1 a 4) para las dos universidades fue de $(x: 3,05)$, la importancia del repositorio institucional $(x: 3,17)$, derechos de autor $(x: 2,92)$, y la escala general $(x: 3,05)$. Lo anterior refleja a nivel general una tendencia positiva hacia la visibilización de la producción científica. Se pueden observar en la tabla II las puntuaciones generales de cada una de las escalas, encontrando que reportan tendencias de distribución normal, sin comportamiento atípico en ninguna categoría.

De otra parte, se estimaron las correlaciones entre las dimensiones particulares de las escalas (Véase tabla III). Cabe resaltar que la correlación entre la escala de visibilización de la producción científica y la importancia del repositorio es de $(r: .265)$ y es estadísticamente significativa al 99\%. La estimada entre derechos de au- tor y visibilización de la producción científica es ( $r$ : 190). Mientras que la correlación más fuerte que se presentó entre las subescalas es entre la importancia del repositorio y derechos de autor con ( $r$ : .462), siendo la mejor magnitud. En todos los casos las correlaciones son positivas lo cual también da cuenta de la consistencia interna entre las escalas. Igualmente, se presentan las correlaciones subescala y escala, para el caso de visibilización de la producción científica con la escala general corresponde a ( $r$ : .637), con la importancia del repositorio ( $r: .790)$, y en relación con los derechos de autor ( $r$ : .770). Estas correlaciones son altas dado que son componentes directos de construcción de la escala.

A continuación, se presenta la comparación de dos muestras independientes para identificar si existen diferencias estadísticamente significativas

Tabla I. Factores que atribuyen los docentes al interés de publicación

\begin{tabular}{|c|c|c|c|}
\hline UNIVERSIDAD & & Frecuencia & Porcentaje \\
\hline \multirow{6}{*}{ Uniguajira } & Realización Personal & 21 & 16,2 \\
\hline & Comunicación Ideas & 30 & 23,1 \\
\hline & Exigencia Laboral & 5 & 3,8 \\
\hline & Responsabilidad Social & 16 & 12,3 \\
\hline & Visibilizar Investigaciones & 58 & 44,6 \\
\hline & Total & 130 & 100,0 \\
\hline \multirow{6}{*}{ UANL } & Realización Personal & 16 & 13,4 \\
\hline & Comunicación Ideas & 8 & 6,7 \\
\hline & Exigencia Laboral & 34 & 28,6 \\
\hline & Responsabilidad Social & 5 & 4,2 \\
\hline & Visibilizar Investigaciones & 56 & 47,1 \\
\hline & Total & 119 & 100,0 \\
\hline
\end{tabular}

Tabla II. Promedio general por categorías de análisis

\begin{tabular}{lcccc}
\hline & $\begin{array}{c}\text { Visibilización Producción } \\
\text { Científica }\end{array}$ & $\begin{array}{c}\text { Importancia } \\
\text { Repositorio }\end{array}$ & $\begin{array}{c}\text { Derechos } \\
\text { Autor }\end{array}$ & $\begin{array}{c}\text { Escala } \\
\text { Visibilidad } \\
\text { Científica }\end{array}$ \\
\hline N $\quad$ Válidos & 249 & 249 & 249 & 249 \\
\hline Perdidos & 0 & 0 & 0 & 0 \\
Media & 3,05 & 3,17 & 2,92 & 3,05 \\
Moda & 3,11 & 3,200 & 2,87 & 3,06 \\
Desv. típ. & 3,22 & 3,20 & 3,00 & 3,06 \\
Asimetría & 0,415 & 0,446 & 0,467 & 0,325 \\
Mínimo & $-0,509$ & $-0,078$ & $-0,046$ & $-0,024$ \\
Máximo & 1,56 & 1,80 & 1,50 & 2,12 \\
Suma & 4,00 & 4,00 & 4,00 & 4,00 \\
\end{tabular}


Tabla III. Correlaciones entre las dimensiones particulares de las escalas

\begin{tabular}{|c|c|c|c|c|c|}
\hline & & $\begin{array}{c}\text { Visibilización } \\
\text { Producción } \\
\text { Científica }\end{array}$ & $\begin{array}{l}\text { Importancia } \\
\text { Repositorio }\end{array}$ & $\begin{array}{l}\text { Derechos } \\
\text { Autor }\end{array}$ & $\begin{array}{c}\text { Escala } \\
\text { Visibilidad } \\
\text { Científica }\end{array}$ \\
\hline \multirow{3}{*}{$\begin{array}{l}\text { Visibilización } \\
\text { Producción Científica }\end{array}$} & C. de Pearson & 1 & $0,265^{* *}$ & $0,190^{* *}$ & $0,637^{* *}$ \\
\hline & Sig. (bilateral) & & 0,000 & 0,003 & 0,000 \\
\hline & $\mathrm{N}$ & 249 & 249 & 249 & 249 \\
\hline \multirow{3}{*}{$\begin{array}{l}\text { Importancia } \\
\text { Repositorio }\end{array}$} & C. de Pearson & $0,265^{* *}$ & 1 & $0,462^{* *}$ & $0,790^{* *}$ \\
\hline & Sig. (bilateral) & 0,000 & & 0,000 & 0,000 \\
\hline & $\mathrm{N}$ & 249 & 249 & 249 & 249 \\
\hline \multirow{3}{*}{ Derechos Autor } & C. de Pearson & $0,190^{* *}$ & $0,462^{* *}$ & 1 & $0,770^{* *}$ \\
\hline & Sig. (bilateral) & 0,003 & 0,000 & & 0,000 \\
\hline & $\mathrm{N}$ & 249 & 249 & 249 & 249 \\
\hline \multirow{3}{*}{$\begin{array}{l}\text { Escala Visibilidad } \\
\text { Científica }\end{array}$} & C. de Pearson & $0,637^{* *}$ & $0,790^{* *}$ & $0,770^{* *}$ & 1 \\
\hline & Sig. (bilateral) & 0,000 & 0,000 & 0,000 & \\
\hline & $\mathrm{N}$ & 249 & 249 & 249 & 249 \\
\hline
\end{tabular}

**. La correlación es significativa al nivel 0,01 (bilateral).

entre los promedios arrojados en cada una de las escalas. Tal como se aprecia en la comparación establecida en la figura 2 . Se identifica que existe una clara tendencia en la cual la UANL tiene mayor nivel de apropiación y de disposición favorable hacia la visibilización de la producción científica. Es de aclarar que esta universidad viene de un proceso más largo frente al uso del repositorio institucional y cuenta con políticas institucionales de acceso abierto al conocimiento. Mientras que para el caso de la Uniguajira apenas es un proceso que se está impulsando en este momento. Cabe resaltar que en todos los casos existieron diferencias estadísticamente significativas en los promedios arrojados siendo superiores para la UANL, con excepción de la importancia del repositorio que es similar con ( $x$ : $3,18)$ para Uniguajira y $(x: 3,17)$ para UANL.

En la figura 2 se presenta un análisis interno de cada una de las universidades por cada subescala, evidenciando como la escala más baja para el caso de Uniguajira la de derechos de autor con ( $x: 2,82)$, luego sigue visibilidad de la producción científica con $(x: 2,92)$, la de mejor valoración fue importancia del repositorio institucional con ( $x$ :

Figura 2. Comparación frente a categorías de análisis de producción científica

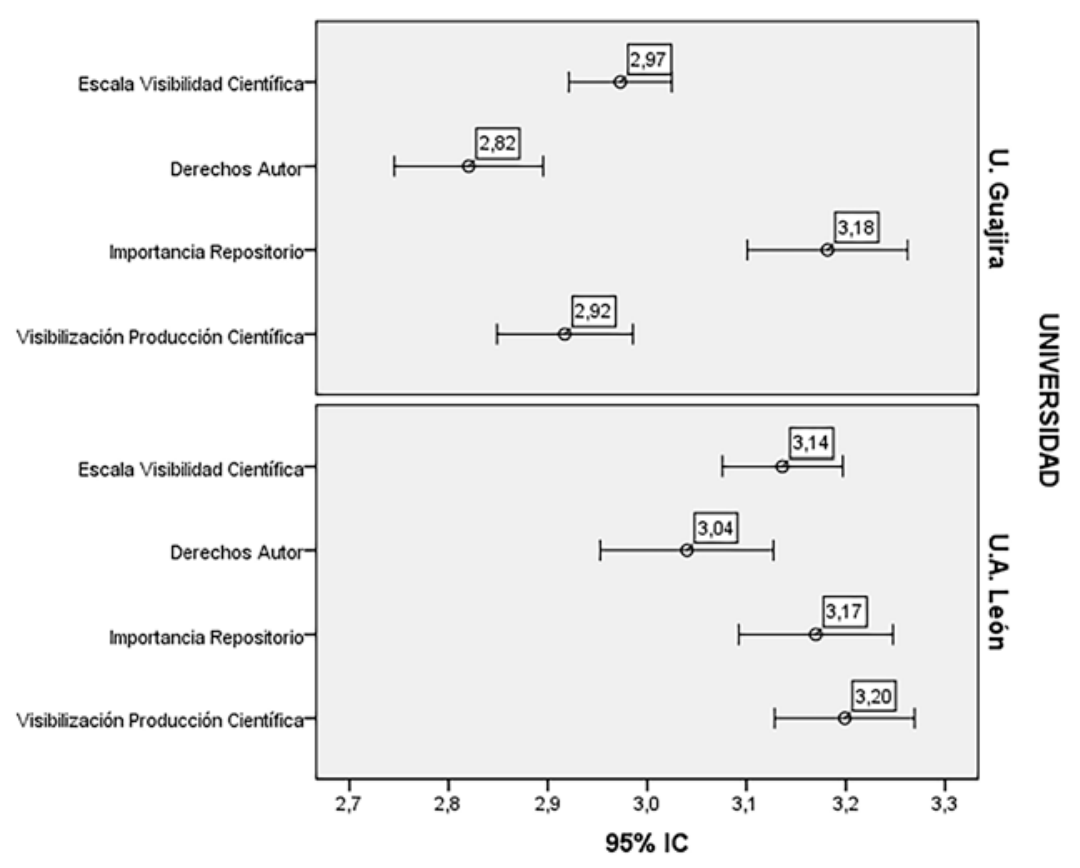


$3.18)$, y para la escala general correspondió a ( $x$ : $2,97)$. Mientras que para el caso de UANL la más baja fue derechos de autor con ( $x$ : 3.04), seguida importancia del repositorio institucional con ( $x$ : $3,17)$, sigue visibilidad de la producción científica con $(x: 3,20)$, con una escala media general de $(x: 3,3)$. Como se puede identificar para el caso de la UANL, todas las subescalas tienen puntuaciones promedio superiores a $(x: 3,0)$, mientras que para Uniguajira la tendencia es de ( $x: 2.9)$.

A continuación se presentan los perfiles discriminados alrededor de cada una de las dimensiones de la escala para las dos universidades, lo cual permite realizar comparaciones de fondo frente a las categorías de análisis. Por ejemplo, en las figuras 3 y 4 se presenta la primera dimensión que está relacionada con visibilización de la producción científica. Se observa tanto para Uniguajira como para UANL que los ítems de menor aprobación, en una escala de 1 a 4, fueron publicar y compartir resultados de investigación que es una exigencia de la universidad, los sistemas de evaluación y medición influyen en el interés de realizar publicaciones. Mientras que las de mayor apropiación para Uniguajira y UANL fueron: "me gustaría conocer resultados de las investigaciones realizadas por las universidades", con ( $x: 3,51)$, y $(x: 3,45)$ respectivamente, $y$ "considero que la universidad debe avanzar en la visibilidad de sus productos", con $(x: 3,55)$ y $(x: 3,41)$.

En segunda estancia, en las figuras 5 y 6 se presenta la categoría importancia del repositorio institucional. Llama la atención que para el caso de Uniguajira, se resalta su impacto en los procesos de apropiación del conocimiento con ( $x$ : $3,46)$ y la posibilidad de visibilizar resultados de investigación con $(x: 3,45)$. Mientras que para UANL, les parece fundamental compartir resultados de manera libre y a través del repositorio institucional con $(x: 3,30)$.

Figura 3. Categoría de análisis Visibilización de la producción científica Uniguajira

\section{UNIVERSIDAD: U. Guajira}

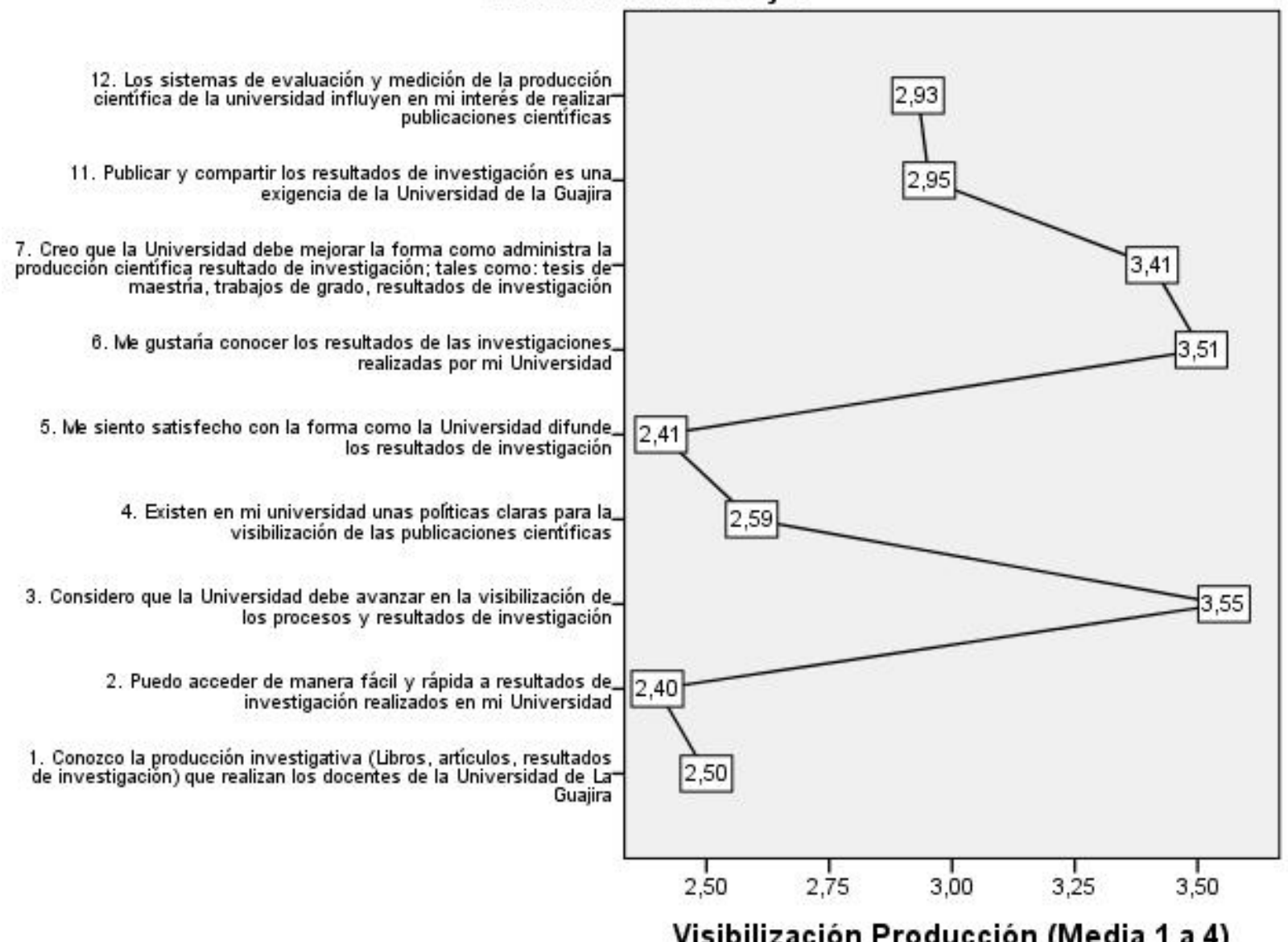

Visibilización Producción (Media 1 a 4) 
Figura 4. Categoría de análisis Visibilización de la producción científica UANL

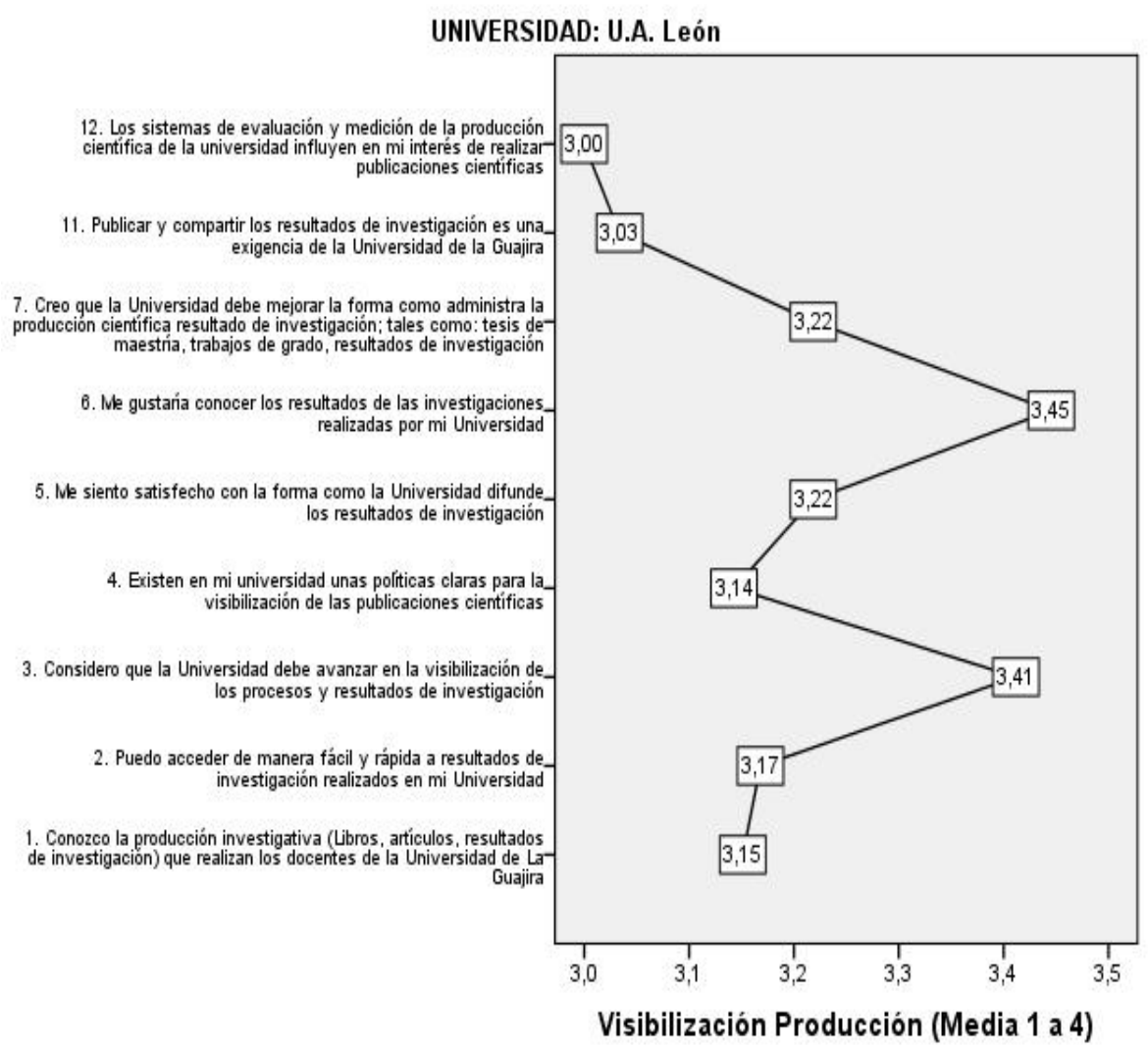

Figura 5. Categoría Importancia del Repositorio Institucional Uniguajira

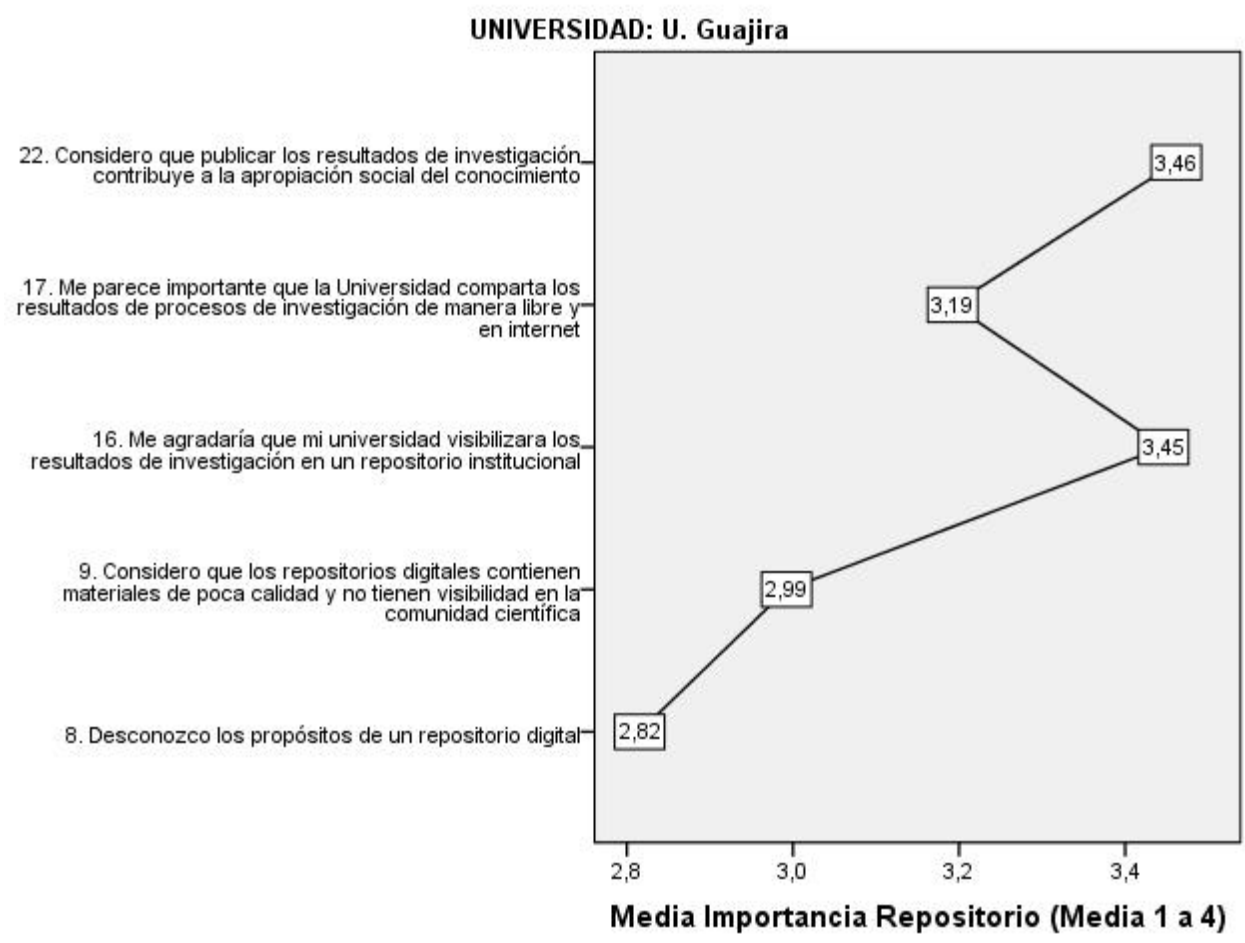


Figura 6. Categoría Importancia del Repositorio Institucional UANL

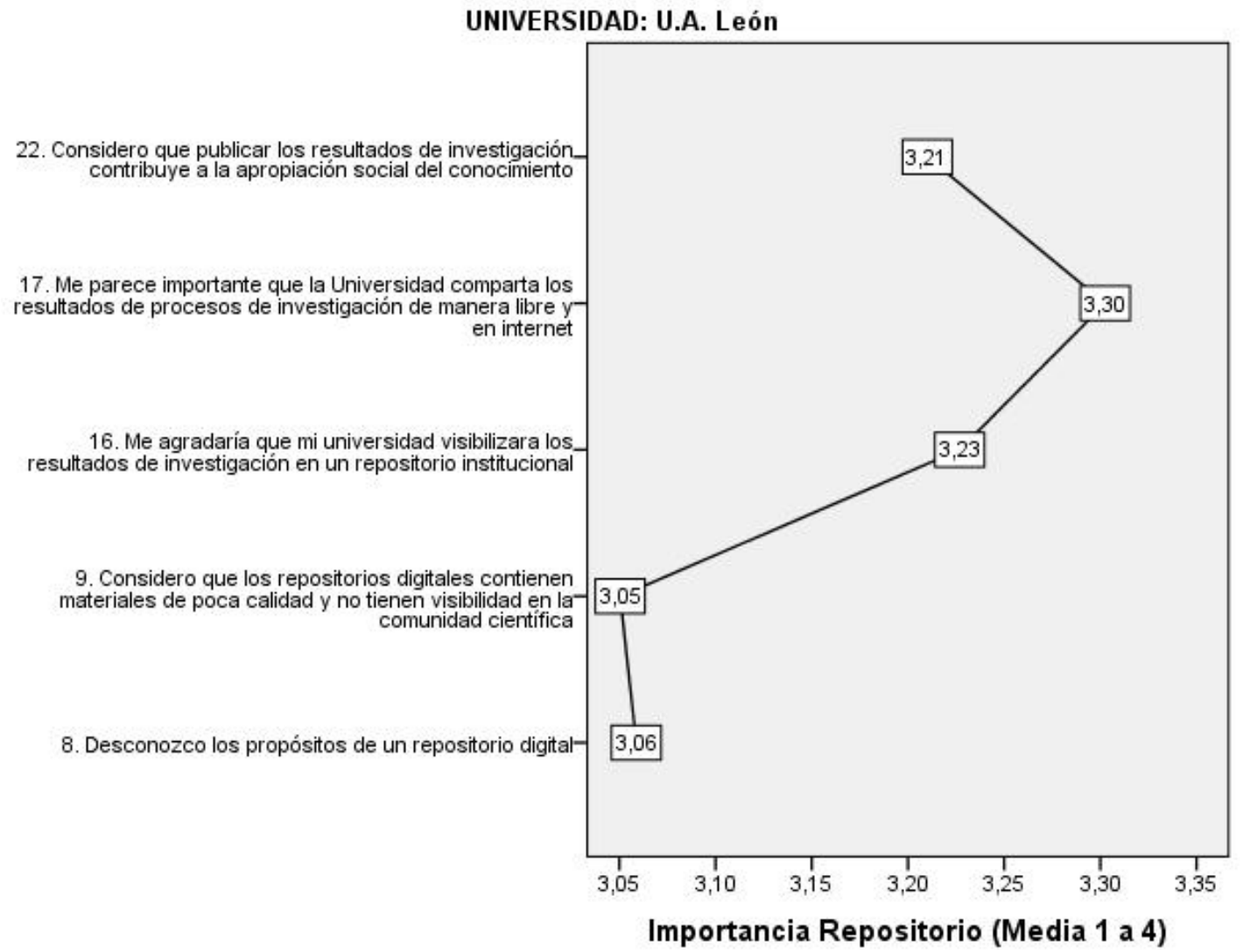

En la categoría derechos de autor, en la figura 7 , se resalta que en Uniguajira el ítem de mayor aprobación fue: "me gustaría tener más facilidad para compartir resultados de investigación", con $(x: 3,39)$, y los de menor aceptación fueron: "siento que es muy complicado realizar publicaciones en revistas indexadas" con ( $x: 2,34)$, "considero que los trabajos tienen mayor impacto cuando se publican de manera libre en internet", con $(x: 2,56)$, y "siento que desconozco los procedimientos para realizar publicaciones científicas" con ( $x$ : 2,65). En la figura 8 se observa que la UANL tuvo mayor aceptación: " me gustaría tener mayor facilidad de compartir resultados de investigación", con ( $x$ : $3,30)$ y "considero que los trabajos tienen mayor impacto cuando se publican de manera abierta y en internet", con $(x: 3,32)$. Las de menor aceptación en UANL coinciden con Uniguajira.

Tal como podemos apreciar en términos generales, las dos universidades comparten un perfil muy parecido en relación con sus tendencias en cada una de las dimensiones. Es claro que UANL tiene puntuaciones más altas que evidencian un índice más favorable frente al proceso, que en parte obedece a que llevan un mayor recorrido con el modelo de la comunicación científica en abierto, y el estímulo a las publicaciones.

\section{DISCUSIÓN}

A partir de los hallazgos identificados en el presente estudio, cabe resaltar que la producción académica y científica en relación con cantidad de publicaciones es superior en la UANL, en comparación con Uniguajira. En este sentido, en lo referente al rango de más de tres publicaciones científicas por docentes, Uniguajira presenta un menor nivel de producción con $17,0 \%$ ante un $73,8 \%$ registrado en la producción de la UANL. Aunque los docentes de las dos universidades concuerdan en la necesidad que existe en educación superior de visibilizar la producción académica y científica, es evidente que la UANL tiene mayores avances en la consolidación de políticas institucionales que impulsan la investigación y la comunicación académica y científica, lo cual redunda en producción de nuevo conocimiento. 
Figura 7. Categoría Derechos de Autor y Acceso Abierto en Uniguajira

\section{UNIVERSIDAD: U. Guajira}

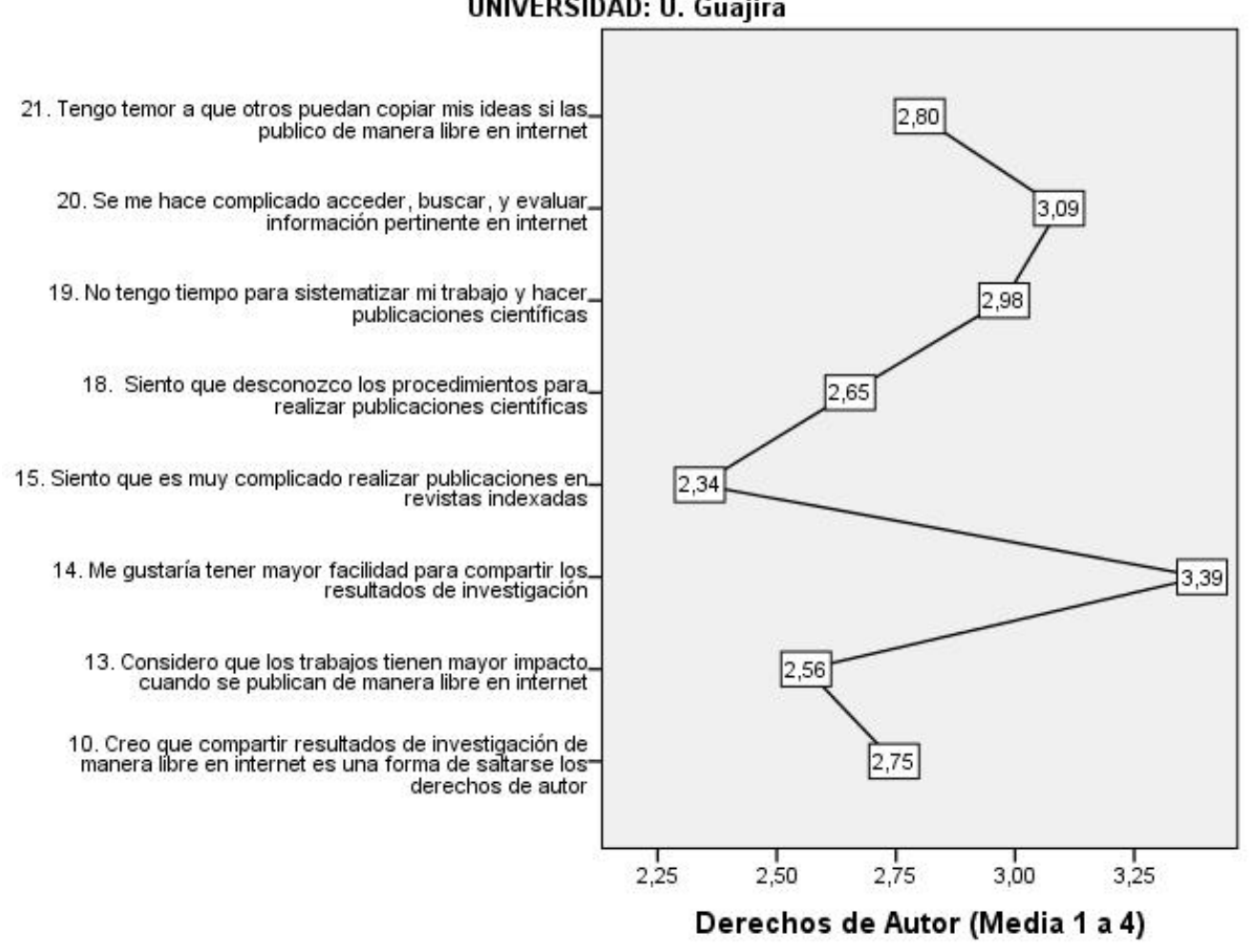

Figura 8. Categoría Derechos de Autor y Acceso Abierto en UANL

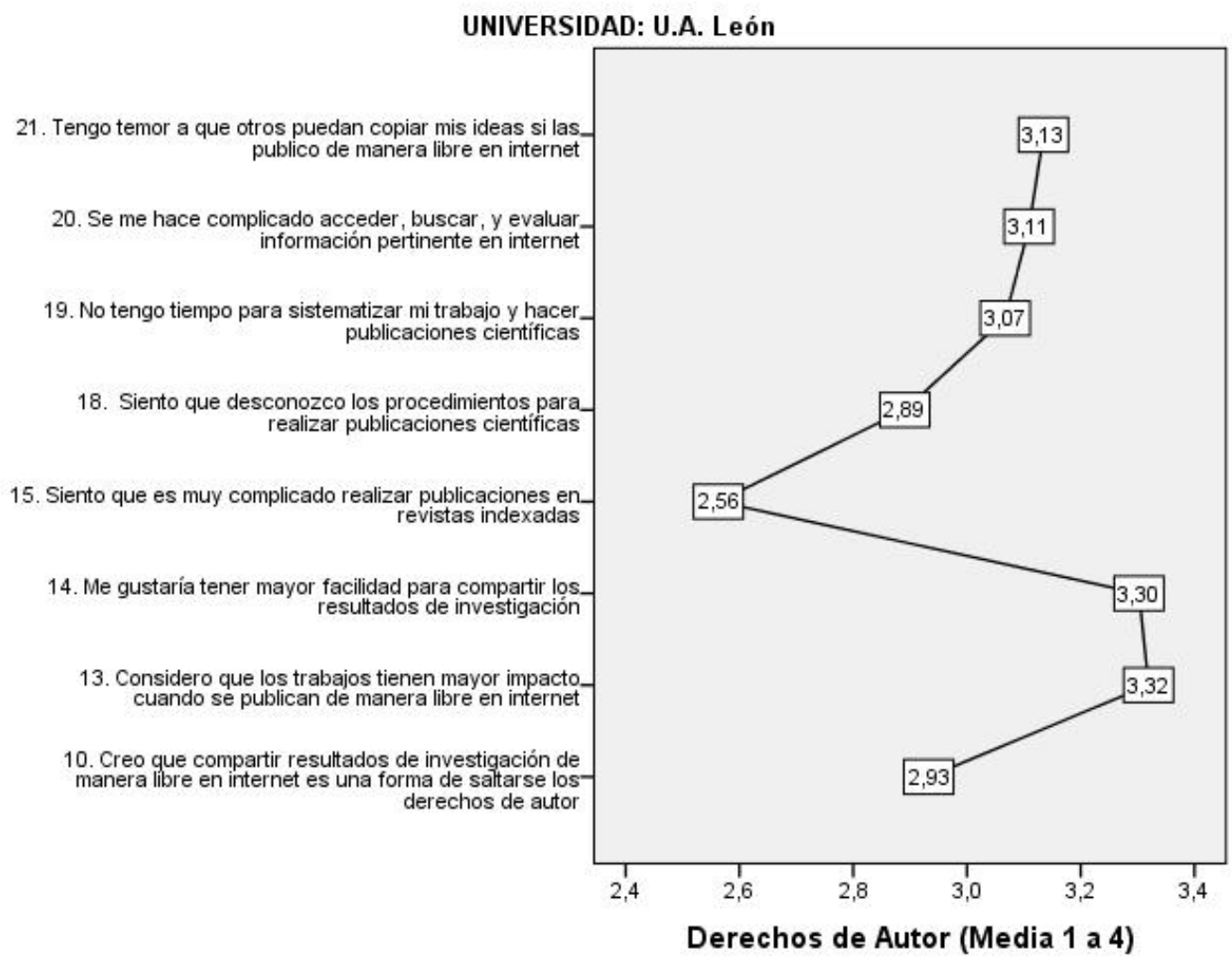


Al respecto, cobra sentido el aporte de Abadal (2013) al considerar que dentro de las ventajas del acceso abierto está el incremento de la investigación, y facilitar la transferencia de conocimiento. Es evidente que los procesos de investigación y comunicación científica en acceso abierto que responden a políticas institucionales en educación superior, juegan un papel estratégico para avanzar en la producción investigativa que tienen las universidades. En este sentido se concuerda con Bongiovani y otros (2017) en que los docentes reconocen los beneficios de la comunicación científica en abierto. Asimismo, Arévalo y otros (2008), Abadal y otros (2013), y Melero y Hernández (2014) consideran necesario que las instituciones incentiven este tipo de políticas. No obstante, las universidades además de promover el uso de recursos de libre acceso, deben fortalecer las capacidades de investigación y publicación de la producción científica (Cortés-Sánchez, 2016; Pérez \& Fuentes, 2016).

En relación a la categoría de análisis Visibilización de la producción científica y académica de la Universidad, llama la atención que en las dos universidades los docentes aseguran que les gustaría conocer resultados de las investigaciones realizadas por la universidad y consideran que la universidad debe avanzar en la visibilidad de sus productos. Es decir, aunque en sus valoraciones las dos universidades guardan la misma tendencia en la importancia que dan a que se desarrollen estrategias de comunicación de resultados de investigación, Uniguajira muestra las valoraciones más bajas frente a satisfacción de los docentes en la forma como la universidad difunde los resultados de investigación y frente a las formas como puede acceder a los mismos.

Se concuerda con Pinto y otros (2017) en que se han generado transformaciones y retos en la educación relacionados con las nuevas dinámicas comunicativas, y con Durat y Mengual-Andrés (2014) en que las TIC han incorporado cambios en la difusión de la ciencia, debido a que han posibilitado otras formas de producción y distribución del conocimiento. De igual manera Abadal y otros (2013) consideran que a nivel universitario se ha avanzado hacia el acceso abierto por las ventajas que ofrece al docente y no como resultado de planes y políticas. No obstante, es necesario incorporar a nivel organizativo políticas que incentiven la investigación y la visibilización de la producción científica en abierto, y a nivel académico se requiere fortalecer las practicas docentes para aprovechar esa disposición que tienen los investigadores de compartir, colaborar, y construir ciencia en la era digital.

Frente a la categoría Importancia del Repositorio Institucional los docentes de las dos uni- versidades aseguran conocer los propósitos de un repositorio digital y la importancia que tiene en la comunicación científica de la institución. De igual manera, creen que los repositorios digitales contienen materiales de calidad y ayudan a la visibilidad en la comunidad académica. Asimismo, para el caso de Uniguajira, donde se está en proceso de consolidación del repositorio institucional, los docentes aseguran desconocer la producción científica que tiene la universidad debido a que no está organizada en un espacio visible y existe desconocimiento del repositorio institucional.

En este sentido, se considera que la tecnología digital y la colaboración académica se han convertido en aliados para abordar problemas de investigación y que los repositorios institucionales son unas bases de datos que permiten administrar la producción científica. Se concuerda con Abadal (2013) y Bongiovani y otros (2017) en que gracias a los estímulos a los autores existe aceptación de los académicos frente al modelo que promueve el acceso libre y la publicación en repositorios y revistas de acceso abierto. De igual manera, la difusión de la producción científica a través de los repositorios da transparencia a la forma de obtención de los datos, permiten reutilizarlos, y validar los resultados (Melero y Hernández, 2014).

En relación con la categoría Derechos de autor y acceso abierto, los docentes aseguran que sus trabajos tienen mayor impacto cuando se comparten de forma libre en internet. No obstante, llama la atención que los participantes creen que compartir resultados de investigación en internet es una forma de saltarse los derechos de autor, el 33\% de los docentes siente temor a perder los derechos sobre su trabajo cuando se comparte en internet, y el $40 \%$ se le hace complicado acceder, buscar y evaluar información disponible en la web. Es decir, aunque el docente reconoce que debe difundir los resultados de investigación, existe desconocimiento frente a los derechos que el autor que tiene sobre la obra y los tipos de licenciamientos que se pueden usar para determinar los usos que quiere dar a su publicación.

Se coincide con Abadal (2013) sobre el desconocimiento de las falsas creencias y malentendidos frente a los derechos de autor en acceso abierto. La comunicación científica en abierto no busca saltarse los derechos de autor, por el contrario, se promueve el uso de licencias libres que pueden condicionar el uso de las publicaciones y exigir el reconocimiento a los autores. Es decir, el acceso abierto busca eliminar las barreras económicas o de derechos de explotación que afectan la utilización del conocimiento, y promueve compartir y reutilizar la comunicación científica de manera responsable y ética (Melero y Hernández, 2014). 
Los hallazgos derivados del presente estudio resaltan la valoración de la importancia que tiene el acceso abierto frente a la visibilización de la producción científica y académica, así como en el correspondiente impacto que tienen las publicaciones científicas dentro de los procesos de consolidación de las redes académicas especializadas, en las cuales se genera una relación sinérgica que contribuye a la generación del nuevo conocimiento unido a los procesos de desarrollo tecnológico soportado con el uso de las TIC y los procesos de innovación y apropiación social del conocimiento (Ausloss, 2013; López-Torres, Guzmán, Castro \& Ramírez, 2016).

\section{CONCLUSIONES}

Es interesante que las dos universidades al compararse generan unos escenarios para entender porque es importante establecer estrategias de comunicación y administración de la producción científica a través de políticas de acceso abierto, y la importancia que tiene el repositorio institucional. Internet posibilita a las instituciones de educación superior participar en una nueva forma de comunicar la ciencia, que rompe con los esquemas tradicionales y que tiene como principales aliados a los docentes. A continuación, se enuncian tres conclusiones en correspondencia con las dimensiones de análisis, orientadas a la comprensión de la percepción del docente universitario sobre la comunicación científica en abierto.

1. Las instituciones de educación superior inciden en la disposición que tienen los docentes para colaborar desde nuevas formas de difusión del conocimiento. El trabajo que vienen desarrollando la UANL y Uniguajira para visibilizar la producción científica y académica desde políticas institucionales de acceso abierto impulsa a los docentes a utilizar plataformas y redes académicas para compartir sus publicaciones y establecer contacto con otros investigadores. De igual manera, son los docentes los que más cuestionan la forma como la institución difunde los resultados de investigación, siendo necesario que el repositorio institucional se convierta en un espacio dinámico que vaya más allá de cumplir la función de archivo digital.

2. Son fundamentales los repositorios institucionales en la administración, conservación y divulgación de los resultados de investigación. Existe disposición por parte de los docentes de las dos universidades por compartir los resultados de investigación a través del repositorio. Aunque hay mayor desconocimiento por parte de Uniguajira de la producción científica de la universidad, se reconoce que el acceso abierto contribuye en mayor medida a la apropiación social del conocimiento. De igual manera, las dos universidades valoran los repositorios institucionales y consideran que se publican materiales de calidad.

3. Existe en los docentes reserva al compartir resultados de investigación en acceso abierto, en tanto hay desconocimiento frente a los derechos que el autor tiene al publicar una obra a través de internet y las formas de determinar los usos que quiere dar a su publicación. No obstante, se reconoce que la tecnología digital se ha convertido en un aliado de las universidades periféricas para visibilizar la producción científica, como es el caso de Uniguajria. En este sentido, se considera que es necesario desarrollar procesos de formación con los docentes para que aclaren los malentendidos frente al acceso abierto, y comprendan que en la sociedad mediada por lo digital, el conocimiento que se comparte de manera libre a través de internet, ayuda a generar comunidades de práctica, y permite visibilizar la producción académica y científica local.

No obstante, este estudio presenta algunas limitaciones relacionadas con conocer niveles de competencia digital, e identificar las redes académicas a las que pertenece el docente que pueden influir en su deseo de compartir resultados de investigación a través de internet. De igual manera, aunque este estudio presenta las edades de los participantes, no se hace una diferenciación de los resultados por cada una de las categorías de análisis, dejando pendiente revisar si las nuevas generaciones de investigadores tienen actitudes más positivas hacia la comunicación científica en abierto.

Finalmente, para estudios futuros se recomienda analizar el impacto de la adopción de las políticas de acceso abierto en la producción y visibilización de la producción científica en universidades periféricas. Es necesario revisar si existen mejoras en los procesos de investigación y estímulo a la publicación, o si sigue aumentando la producción de las universidades que tradicionalmente han desarrollado procesos de investigación, y las pequeñas universidades continúan rezagadas. De igual manera, se deben estudiar las acciones de política encaminadas a que la filosofía adoptada por la institución frente al acceso abierto, se conozca y se practique por los principales usuarios.

\section{AGRADECIMIENTOS}

Este trabajo ha sido financiado por la Universidad de La Guajira (Colombia) y Universidad Autónoma de Nuevo León (México). Agradecimiento especial a los profesores Mayerlin Mejía Pareja y José María Mejía Caballero por su contribución en el proceso de investigación. 


\section{ACKNOWLEDGEMENTS}

This work has been funded by the Universidad de La Guajira (Colombia) and Universidad Autónoma de Nuevo León (México). Special thanks to professors Mayerlin Mejía Pareja and José María Mejía Caballero for their contribution to the research process.

\section{REFERENCIAS}

Abadal, E. (2013). Acceso abierto a la ciencia. Barcelona: Editorial UOC. (Colección el profesional de la información). Disponible en: http://eprints.rclis. org/16863/1/2012-acceso-abierto-epi-uoc-vfinalautor.pdf [Fecha de consulta: 16/06/2017].

Abadal, E.; Ollé-Castellà, C.; Abad-García, F.; Melero, R. (2013). Políticas de acceso abierto a la ciencia en las universidades españolas. Revista Española de Documentación Científica, 36 (2): e007. http:// dx.doi.org/10.3989/redc.2013.2.933

Aguado-López, E.; Becerril-García, A. (2016). ¿Publicar o perecer? El caso de las Ciencias Sociales y las Humanidades en Latinoamérica. Revista Española de Documentación Científica, 39(4), e151. http:// dx.doi.org/10.3989/redc.2016.4.1356

Arencibia, R. (2006). Las iniciativas para el acceso abierto a la información científica en el contexto de la web semántica. Biblios, 7 (25-26), 1-14.

Arévalo, J.; Subirats, I.; Martínez, M. (2008). Informe APEI sobre acceso abierto. Gijón: APEI, Asociación Profesional de Especialistas en Información.

Ausloos, M. (2013). A scientometrics law about coauthors and their ranking: the co-author core. Scientometrics, 95(3), 895-909. https://doi. org/10.1007/s11192-012-0936-x

Bongiovani, P.; Miguel, S.; Hernández-Pérez, T. (2017). Actitudes y percepciones de los evaluadores de la carrera científica en Argentina sobre la publicación en acceso abierto. Revista Española de Documentación Científica, 40(2), e171. http:// dx.doi.org/10.3989/redc.2017.2.1404

Cabrera, K. I. (2014). Modelos de acceso abierto en educación y ciencia, Educación y Educadores, 17 (2), pp. 321-338. Cundinamarca, Colombia: Universidad de La Sabana.

Canessa, E. y Zennaro, M. (2008). Difusión científica y las iniciativas de acceso abierto. Trieste, Italia: Centro Internacional de Física Teórica Abdus Salam. Disponible en http://eprints.rclis.org/13661/1/AccesoAbiertoConocimientoP.pdf [Fecha de consulta: 06/03/2018].

Cortés, O.; Pinto, A.; Atrio, S. (2015). E-portafolio como herramienta construccionista del aprendizaje activo en tecnología educativa. Revista Lasallista de Investigación, 11(2), 36-44. https://doi. org/10.22507/rli.v12n2a4

Cortés-Sánchez, J. (2016). Eficiencia en el uso de bases de datos digitales para la producción científica en universidades de Colombia. Revista Española de Documentación Científica, 39(2), e130. http:// dx.doi.org/10.3989/redc.2016.2.1320
Creative Commons Colombia (2016). Sobre las licencias Creative Commons. Disponible en: http:// co.creativecommons.org/?page_id $=13 \quad$ [Fecha de consulta: 15/06/2017].

Declaración de Bethesda (2003). Bethesda Statement on Open Access Publishing. Disponible en: https:// bit.ly/2fuDVrL [Fecha de consulta: 11/01/2017].

Declaración de Berlín (2003). Declaración de Berlín sobre Acceso Abierto al Conocimiento en Ciencias y Humanidades. Disponible en: https://www.um.es/c/ document_library/get_file?uuid =f3736570-bb8440b3-8a2e-a9397ef7ef30\&groupId $=793464$ [Fecha de consulta: 10/01/2017].

Declaración de Budapest (2002). Iniciativa de Budapest para el Acceso Abierto. Disponible en: http://www. budapestopenaccessinitiative.org/translations/spanish-translation [Fecha de consulta: 13/01/2017].

Durat, J.; Mengual-Andrés, S. (2014). Impacto de la Sociedad del Conocimiento en la universidad y en la comunicación científica. RELIEVE, 20 (2), art. M4 https://doi.org/10.7203/relieve.20.2.4343

González-Alcaide, G.; Gómez-Ferri, J. (2014). La colaboración científica: principales líneas de investigación y retos de futuro. Revista Española de Documentación Científica, 37(4): e062. https://doi. org/10.3989/redc.2014.4.1186

Guédon, J. (2011). El acceso abierto y la división entre ciencia "principal" y "periférica". Crítica y Emancipación (6): 135-180. http://biblioteca.clacso.edu.ar/ar/libros/secret/CyE/6/CyE6_Guedon-CLACSO.pdf [Fecha de consulta: $18 / 03 / 2017]$

León, O.; Montero, I. (2003). Métodos de investigación en psicología y educación. Madrid: McGraw-Hill Interamericana.

López-Torres, G. C.; Guzmán, G. M.; Castro, S. Y. P.; Ramírez, R. G. (2016). Collaboration and innovation activities in SMEs. Contaduría y Administración, 61(3).

Melero, R.; Hernández-San-Miguel, J. (2014). Acceso abierto a los datos de investigación, una vía hacia la colaboración científica. Revista Española de Documentación Científica, 37(4), e066. https://doi. org/10.3989/redc.2014.4.1154

Pérez, O.; Fuentes, E. (2016). Clasificación de la actividad científica estudiantil en la educación médica superior. Educación Médica, 17(2), 55-60. https:// doi.org/10.1016/j.edumed.2016.02.001

Pinto, A.; Cortés, O.; Alfaro, C. (2017). Hacia la transformación de la práctica docente: modelo espiral de competencias TICTACTEP. Píxel-Bit. Revista de Me- 
dios y Educación, no 51. https://doi.org/10.12795/ pixelbit.2017.i51.03

Pinto, A.; Villanueva, C. (2016). Escala actitudinal de situaciones asociadas a la visibilización de la producción científica. Disponible en http://repositoryinst. uniguajira.edu.co/xmlui/handle/123456789/87 [Fecha de consulta: 13/03/2018].

Pinto, A.; Mejía, M.; Mejía, J. (2017). Políticas y prácticas de acceso abierto en la Uniguajira. Riohacha: Uniguajira. Disponible en http://repositoryinst.uniguajira.edu.co/xmlui/handle/123456789/78 [Fecha de consulta: 28/11/2017].

Rio Romero, L. del (2014). Integración del Sistema de Gestión de la Investigación (CRIS) con un Repositorio Institucional. El modelo de la Universidad Carlos III de Madrid. (Tesis inédita de maestría). Madrid: Universidad Carlos III de Madrid.

Serna-Saucedo, N.; Villanueva-Valadez, C. (2014). Implementación del acceso abierto al conocimiento y repositorio institucional UANL. Disponible en: http://eprints.uanl.mx/4062/ [Fecha de consulta: 23/02/2017].

Suber, P. (2015). Acceso Abierto (Primera edición en Español). Toluca, Estado de México: Universidad Autónoma del Estado de México.

Swan, A. (2013). Directrices para políticas de desarrollo y promoción del acceso abierto. Paris: Unesco.

Valenzuela, J. R.; Flores, M. (2012). Fundamentos de Investigación Educativa. Monterrey, México: Editorial Digital del Tecnológico de Monterrey.

Valverde, J. (2013). El acceso abierto al conocimiento científico. Disponible en: http://reunid.eu/publicaciones/el-acceso-abierto-al-conocimiento-cientifico/ [Fecha de consulta: 05/04/2017].

Vargas, E. (2014). La política editorial de acceso abierto: horizontes de la democratización del conocimiento para el desarrollo. Disponible en: https://dialnet. unirioja.es/servlet/articulo?codigo $=5166474 \quad[\mathrm{Fe}-$ cha de consulta: 28/02/2017]. 